\title{
Le interviste INN-possibili: CKD-MBD
}

La rubrica Le Interviste INN-possibili per tutto il 2010 sarà incentrata su un argomento che "tira molto" la CKD-MBD.

Alcuni tra i più autorevoli cultori dell'argomento hanno accettato di rispondere ad un questionario composto da domande uguali per tutti (pur in considerazioni delle diverse realtà specifiche), da un gruppo di 3-4 domande scelte tra altre 14. Ho lasciato anche facoltà di proporre domande e risposte personalizzate, e a chi lo ritenesse opportuno di lasciare al lettore una nota conclusiva in approfondimento ai quesiti o per l'argomentazione più sentita dall'Autore stesso.

È questo un modo poco ortodosso, ma spero sufficientemente originale, per attrarre l'attenzione del lettore su un argomento complesso, articolatissimo e in continua evoluzione, permeato da fini teorie che necessitano di tempi per fortuna sempre più brevi per vederle concretizzarsi. Ed allora quale modo migliore per esporre teoria in pratica se non con quesiti mirati a cultori differenti ma sicuramente tutti in continua full-immersion nell'argomento?

Il mio ringraziamento a tutti i colleghi che ancora una volta si sono prestati nonostante i molteplici impegni e mi/ci hanno onorato con le loro dotte disquisizioni. Una buona, proficua e divertente lettura a tutti. 


\section{Intervista a Mario Cozzolino}

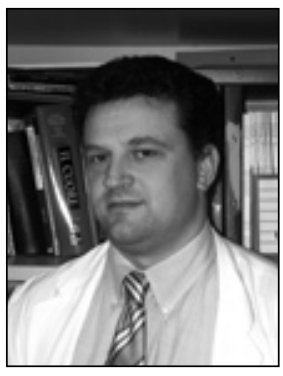

Dr. Mario Cozzolino

UO Nefrologia e Dialisi

AO San Paolo

Polo Universitario, Milano

mariocozzolino@hotmail.com

Nel centro ove presti attività qual è la percentuale di pazienti monitorati per CKD-MBD stadio 3-5 e quali marker biologici usi a tal fine?

Nel mese di agosto 2009 sono state pubblicate su un numero dedicato di Kidney International le nuove linee guida KDIGO (Kidney Disease Improving Global Outcomes) per la diagnosi, prevenzione e trattamento della CKD-MBD (Chronic Kidney Disease - Mineral and Bone Disorder), attraverso la National Kidney Foundation. Lo scopo di queste nuove linee guida è quello di assistere il nefrologo nella cura dei soggetti sia in età adulta sia in età pediatrica, affetti da CKD-MBD, agli stadi della CKD 3-5 non in dialisi, in dialisi, o trapiantati di rene.

Nella nostra Unità Operativa di Nefrologia e Dialisi tutti i pazienti con GFR $<60 \mathrm{~mL} / \mathrm{min}$ vengono monitorati per calcemia totale e ionizzata, fosforemia, PTH intatto, fosfatasi alcalina al momento della prima visita. Quindi, tutti i pazienti con CKD allo stadio 3 sono potenzialmente affetti da CKDMBD. Il calcio e il fosforo vengono poi analizzati a ogni visita successiva (in media 2-3 volte all'anno). Il PTH e la fosfatasi alcalina 1-2 volte all'anno, in considerazione anche del trend dei valori biochimici e della terapia instaurata.

Nel centro ove presti attività pratichi il dosaggio della $25(\mathrm{OH}) \mathrm{D}$ e/o della $1,25(\mathrm{OH})_{2} \mathrm{D} 3$ ?

Se si, in quale tipo di paziente e in quale percentuale? Se no, perché ritieni di non doverla praticare?

Dal mese di aprile 2009 nella nostra Azienda Ospedaliera è possibile dosare i livelli di $25(\mathrm{OH}) \mathrm{D}$. Da allora tutti i pazienti agli stadi della CKD 3-5 non in dialisi, in dialisi, o trapiantati di rene vengono analizzati per i livelli di 25D per 2 volte all'anno, e più precisamente all'inizio della primavera (aprile) e all'inizio dell'autunno (ottobre). Non riteniamo invece utile eseguire di routine l'analisi dei livelli di 1,25(OH)D3, che viene eseguita solo in casi selezionati.
Applichi la correzione di 25(OH)D in caso di carenza? Perché, con quale protocollo e in quale tipo di paziente?

Una delle condizioni che può essere alla base dell'aumentata sintesi di PTH nei pazienti con CKD-MBD è la carenza di $25(\mathrm{OH}) \mathrm{D}$, che però può essere presente anche in pazienti con funzione renale normale. La valutazione preliminare dei livelli sierici della 25-idrossivitamina D e l'eventuale conseguente loro correzione avviene mediante l'utilizzo dell'ergocalciferolo o del colecalciferolo, per ridurre i livelli di PTH e promuovere il fronte di mineralizzazione osseo e la deposizione di calcio nelle ossa.

Nel centro ove presti attività in quale percentuale o in quali casi clinici particolari pratichi la biopsia ossea?

Le nuove linee guida KDIGO contengono raccomandazioni su come valutare e trattare le alterazioni del metabolismo minerale. Le KDIGO suggeriscono quali parametri biochimici considerare, quando eseguire una agobiopsia ossea, come e quando valutare le complicanze cardio-vascolari in questi pazienti. Inoltre, vengono illustrati i trattamenti per la cura dell'iperfosforemia, delliperparatiroidismo e della patologia osteo-scheletrica nei pazienti con CKD allo stadio $3-5$, in dialisi e non. In particolare, non si pratica l'agobiopsia ossea se non per casi eccezionali.

Per poter leggere le linee guida KDIGO è necessario ricordare che già nel 2006 Sharon Moe et al (2) avevano definito la CKD-MBD come una patologia sistemica del metabolismo minerale e osseo causata dalla $\mathrm{CKD}$, che si può presentare con uno o più dei seguenti segni o sintomi:

- anormalità biochimiche (alterato metabolismo del calcio, fosforo, PTH o vitamina D);

- anormalità del turnover, mineralizzazione, volume, crescita lineare o forza ossea;

- calcificazioni vascolari o extra-scheletriche.

In tale ambito la definizione "classica" di osteodistrofia renale rimane un'alterazione della morfologia ossea nei pazienti con CKD-MBD, misurabile solo con l'istomorfometria ossea, e pertanto con l'agobiopsia ossea.

Nel centro ove presti attività in quale percentuale ed in quali stadi di CKD usi indagini strumentali per la ricerca delle calcificazioni vascolari? Quali indagini utilizzi?

È importante sottolineare il fatto che la stesura delle 
KDIGO Guidelines ha seguito l'approccio metodologico della medicina basata sull'evidenza, trattando quindi metanalisi, review sistematiche e trial clinici randomizzati. Da tale analisi emerge che il paziente con CKDMBD affetto da calcificazioni vascolari e/o valvolari risulta essere ad alto rischio per malattia cardio-vascolare. Pertanto, le calcificazioni vascolari vanno indagate. Nel nostro centro utilizziamo l'ecocardiogrammacolor-Doppler per la valutazione delle calcificazioni valvolari mitralica e aortica, con controllo annuale per tutti i pazienti con CKD-MBD. Per lo studio delle calcificazioni vascolari utilizziamo la radiografia in laterale dell'aorta addominale, con controllo annuale.

Nel centro ove presti attività quale è la percentuale di pazienti in trattamento sostitutivo che resta nel range di normalità (secondo le linee guida SIN) per Ca, Pe PTH?

Ogni capitolo del Supplemento di Kidney International contiene raccomandazioni "graduate" come livello 1 o 2, e dalla qualità dell'evidenza a supporto $(\mathrm{A}, \mathrm{B}, \mathrm{C}, \mathrm{o} \mathrm{D})$. Inoltre, gli Autori hanno potuto aggiungere dei consigli "nongraduati". Il "livello 1" è definito come "raccomandazione", mentre il "livello 2" come "suggerimento". Il grado "A" è elevato, il "B" è moderato, il grado " $\mathrm{C}$ " è basso, mentre il grado " $\mathrm{D}$ " è molto basso.

Se consideriamo le linee guida della SIN, che poi si rifanno alle KDOQI del 2003, nel nostro centro abbiamo circa il 35\% dei pazienti in range per il PTH in peritoneale e in emodialisi, il $55 \%$ per il fosforo e il $65 \%$ per il calcio. Non abbiamo i dati per i pazienti in predialisi.

Nel centro ove presti attività quale è la percentuale dei vari chelanti usati per ricercare il controllo del Ca-P nei pazienti in trattamento sostitutivo (anche in off-label treatment)?

Nel nostro centro utilizziamo il calcio carbonato da solo o in associazione nel $50 \%$ circa dei pazienti, con un dosaggio massimo di 2 grammi al giorno. Il Sevelamer in circa il $45 \%$ dei pazienti. Il Lantanio carbonato nel $20 \%$ circa dei pazienti. L'idrossido di alluminio nel 5\% circa dei pazienti.

Nel centro ove presti attività qual è la percentuale di pazienti in CKD 3-5 sottoposta a dieta ipoproteica per il controllo della iperfosforemia?

Abbiamo circa il 15\% dei pazienti che seguono una dieta ipoproteica per il controllo dell'iperfosforemia.

Nel centro ove presti attività quale percentuale di pazienti in trattamento sostitutivo assumono vit-D o analoghi, calciomimetico, o entrambi?

Nel nostro centro utilizziamo il calcitriolo orale o il paracalcitolo orale o endovena nel 70\% circa dei pazienti in emodialisi o peritoneale. Il calciomimetico sempre in associazione con vitamina $\mathrm{D}$ nel 30\% circa dei pazienti.

PTH e CKD-MBD: quanto artefice o quanto vittima? Ad esempio la carenza di vitamina $D$ (o meglio la riduzione dell'attivazione dei suoi recettori nucleari) è sicuramente un evento che precede l'iperparatiroidismo secondario. Quale di questi due giocatori è in "fuori gioco" per lo sviluppo della patologia ossea dell'uremico e a quale dei due, se possibile, si può imputare un predominio sullo sviluppo di CKD-MBD?

Una delle complicanze più frequenti in corso di malattia renale cronica $(\mathrm{CKD})$ allo stadio 3 e 4 è rappresentata dalliperparatiroidismo secondario (IPTS). Il trattamento classico dell'IPTS, nei pazienti affetti da CKD, con calcitriolo e chelanti del fosforo a base di sali di calcio ha portato ad aumento della deposizione di calcio-fosfato nei tessuti molli. Per i pazienti in dialisi, studi recenti hanno documentato come i nuovi chelanti del fosforo non contenenti né calcio né alluminio (Sevelamer, Lantanio carbonato), gli attivatori selettivi del recettore della vitamina D, VDRAs (paracalcitolo), meno ipercalcemizzanti e iperfosforemizzanti, e i calciomimetici (Cinacalcet) siano efficaci nel trattamento dell'IPTS e potenzialmente utili nella prevenzione dei processi di calcificazione extrascheletrica. È noto che quando decresce il filtrato glomerulare (GFR), si riduce anche l'1alfa idrossilasi renale e di conseguenza diminuiscono i livelli sierici di 1,25-diidrossivitamina D. Questo evento si osserva piuttosto precocemente, a partire dallo stadio 2 della CKD (GFR 60-90 mL/min), quando ancora non si sono verificate variazioni dei livelli di fosforemia e calcemia (14). A mano a mano che l'insufficienza renale progredisce, aumenta il numero di pazienti con bassi livelli di calcitriolo circolanti ed elevati livelli di PTH. Il deficit di calcitriolo innesca 2 eventi principali: 1) la riduzione dell'assorbimento intestinale di calcio con conseguente ipocalcemia; e 2) un ridotto controllo genomico sul prepro-PTHmRNA; entrambi gli eventi rappresentano uno stimolo importante alla sintesi e secrezione del PTH. Inoltre, negli stadi 3 e 4 della CKD esiste la peggiore combinazione di eventi poiché gli aumentati livelli di fosforo sierici possono essere un ulteriore stimolo per la secrezione di PTH, indipendentemente dall'effetto dei bassi livelli di vitamina $\mathrm{D}$.

Del cosiddetto effetto "pleiotropico" della vit-D e dei suoi analoghi, quanto e cosa è applicabile alla CKD-MBD? 
La Vitamina D è oggi considerata un vero e proprio ormone steroideo che si lega con il proprio recettore intracellulare così da produrre una differente attivazione genica da parte dei diversi attivatori, configurando in questo modo il concetto di selettività. A questo riguardo, numerosi sono gli studi epidemiologici e sperimentali che, inizialmente, hanno associato i ridotti livelli di 25OHD e Calcitriolo con il rischio cardio-vascolare, la resistenza insulinica, lo stato diabetico, la produzione di renina, l'ipertensione arteriosa e la proteinuria. Tra i vari effetti favorevoli della Vitamina D sui fattori di rischio cardio-vascolare, certamente i più interessanti sono quelli che condizionano lo sviluppo di calcificazioni vascolari e l'aterosclerosi. È noto infatti come l'attivazione dei VDR sia in grado di bloccare la sintesi del fattore di mineralizzazione noto come Cbfa1 ( RUNX2 ), qualora venga impiegato il paracalcitolo, così come il BMP-2, il collagene tipo I, l'interleukina 1- $\beta$, IL-6 e il TGF-alfa. Tali favorevoli effetti si sommano inoltre alla stimolazione della sintesi degli inibitori della mineralizzazione dei tessuti molli, quali l'Osteopontina e la Gla-protein.

Altri potenziali meccanismi possibilmente dovuti all'attivazione dei VDR sono correlati al controllo dell'asse Renina-Angiotensina-Aldosterone (RAA). È noto infatti come la mancata o ridotta attivazione dei VDR si accompagni ad una maggior attivazione del sistema RAA, con aumento della pressione arteriosa e della massa ventricolare sinistra, per ipertrofia del cardiomiocita come dimostrato nel topo VDR -/-. Per contro, il trattamento con attivatori VDR riporta in condizioni ottimali l'espressione di renina, i livelli di pressione arteriosa e l'ipertrofia miocardica.

\section{Perché supplementare con calcifediolo e calcitriolo $i$ pazienti in CKD 3-5, con carenza di 25(OH)D?}

La carenza di Vitamina D è assai comune sia nella popolazione generale e particolarmente tra i soggetti uremici. Più precisamente, bassi livelli di 25OHD3 sierici sono osservabili in più parti delle regioni degli Stati Uniti, nel 71\% dei pazienti con IRC allo stadio 3 e più marcatamente (83\%) dei pazienti allo stadio 4 dell'insufficienza renale cronica. Nei soggetti sottoposti a trattamento dialitico la quota di pazienti con livelli non adeguati di 25OHD3 sono la quasi totalità. Considerando pertanto che la larga parte di pazienti sono carenti di 25OHD3, ne deriva l'ovvia conclusione che l'attivazione dei VDR dovrebbe essere garantita a tutti i pazienti. Tuttavia è necessario considerare una doppia opzione: la terapia sostitutiva ha significato di recupero di una condizione nutrizionale e pertanto è suggeribile un intervento con Vitamina $\mathrm{D}$ nativa 0 Calcidiolo (25OHD3).
La vera attivazione dei VDR, che può essere considerata secondo le linee guida KDIGO per il controllo della secrezione del PTH, può avvenire col semplice Calcitriolo o con analoghi selettivi quali il Paracalcitolo in considerazione del miglior profilo sierico di calcemia e fosforemia.

Fosforo chiama "ormone"! Rispondono PTH, FGF23 e Klotho mentre $1,25(\mathrm{OH})_{2} D 3$ resta a guardare. Una relazione pericolosa? Più di un triangolo (fisiopatologico)? Un paradigma o cosa?

Tra le fosfatonine, in particolare, l'FGF23, prodotto dagli osteoblasti, è un fattore circolante che agisce come un ormone deputato a mantenere l'omeostasi del fosforo mediante un'azione fosfaturica sul tubulo renale e un'inibizione della 1-alfa-idrossilasi renale che riduce i livelli di calcitriolo. Recentemente l'FGF23 ha ricevuto notevole attenzione non tanto per il suo ruolo nell'omeostasi del fosfato quanto per il suo peculiare legame con un gene anti invecchiamento, chiamato Klotho. Sia Klotho che FGF23 agiscono sulla sintesi della vitamina D e sulla secrezione di PTH.

\section{Commento conclusivo}

I pazienti affetti da insufficienza renale cronica (CKD stadio 3-5) spesso hanno livelli sierici di fosforo elevati, per una riduzione della fosfaturia da parte del rene malfunzionante. Nel corso degli ultimi vent'anni diversi meccanismi ormonali sono stati proposti per spiegare la regolazione dell'omeostasi del fosforo. Nonostante ciò la conoscenza di questo complesso processo fisiopatologico rimane ancora incompleta. L'ormone paratiroideo (PTH) ha un'azione molto efficace nel ridurre il riassorbimento tubulare di fosforo, al fine di contrastare la tendenza all'iperfosforemia in corso di CKD. D'altra parte lo stesso PTH aumenta la sintesi renale di calcitriolo con conseguente aumento dell'assorbimento gastroenterico e renale di fosforo.

Più recentemente, sono state studiate nuove molecole che intervengono nella fisiologia dell'omeostasi del fosforo e nella patogenesi dell'iperparatiroidismo secondario in corso di CKD. Tra questi forse quello che sembra rivestire maggiore interesse è Fibroblast Growth Factor 23 (FGF-23) che sembrerebbe rappresentare un fattore centrale nella patogenesi dell'iperparatiroidismo in corso di CKD. Diversi lavori pubblicati negli ultimi 5 anni hanno portato a nuove conoscenze sul ruolo del FGF-23 nei pazienti con $\mathrm{CKD}$. In maniera molto elegante, BenDov et al (1) hanno mostrato l'esistenza di un nuovo asse 
endocrinologico nelle interazioni fisiologiche tra ghiandole paratiroidee, rene e tessuto osseo nell'omeostasi del fosforo. Infatti, gli Autori hanno documentato per la prima volta in letteratura che FGF-23 oltre a ridurre i livelli sierici di calcitriolo, riduce anche la sintesi (mRNA) e la secrezione di PTH. Poiché FGF-23 è prodotto dagli osteociti e controlla alcune azioni ormonali a livello renale, il nuovo asse endocrino è stato denominato: asse osso-rene ("the bone-kidney axis").

Il meccanismo di azione dell'FGF-23 non è ancora completamente noto, ma sembra certo che agisca attraverso un recettore specifico (FGF-R), espresso a livello renale, paratiroideo, del plesso coroide e ipofisario. Tuttavia l'affinità di FGF-23 per il suo recettore è piuttosto bassa e perché si abbia un significativo effetto biologico, è necessaria la presenza di un cofattore, oggi identificato nella proteina Klotho (2). Gli effetti di questa proteina non sono ancora completamente definiti, ma si ritiene che sia implicata nei processi di invecchiamento.

Nei soggetti con funzione renale normale, FGF-23 riduce la fosforemia attraverso la riduzione del riassorbimento tubulare e l'assorbimento intestinale di fosforo. I livelli sierici di FGF-23 sono aumentati in corso di CKD. Recentemente, è stato pubblicato un lavoro molto interessante su New England Journal Medicine dal gruppo di Harvard (Boston), coordinato da Ravi Thadani e da Myles Wolf (3). In particolare, gli Autori hanno ipotizza- to un'associazione tra i livelli di FGF-23 e la mortalità nei pazienti che iniziano il trattamento emodialitico.

Lo scopo dello studio di Gutierrez et al è stato di osservare l'effetto di elevati livelli di FGF-23 sulla mortalità in pazienti che iniziavano la dialisi. In effetti, gli Autori dimostrano che i livelli aumentati di FGF-23 erano indipendentemente associati con la mortalità nei pazienti che iniziavano il trattamento emodialitico.

In conclusione, la conoscenza di nuovi fattori coinvolti nella fisiopatologia dell'iperparatiroidismo secondario in corso di CKD, come l'FGF-23, rappresenta un punto cruciale per poter migliorare l'approccio clinico, diagnostico e terapeutico di questa patologia.

\section{Bibliografia}

Ben-Dov IZ, Galitzer H, Lavi-Moshayoff V, et al. The parathyroid is a target organ for FGF23 in rats. J Clin Invest 2007; 117(12): 4003-8.

Kuro-o M, Matsumura Y, Aizawa H, et al. Mutation of the mouse klotho gene leads to a syndrome resembling ageing. Nature 1997; 390(6655): 45-51.

Gutierrez OM, Mannstadt M, Isakova T, et al. Fibroblast Growth Factor 23 an mortality among patients undergoing hemodialysis. N Engl J Med 2008; 359: 584-92.

\section{Intervista a Fabio Malberti}

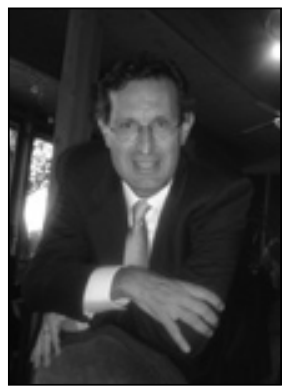

Dr. Fabio Malberti

Divisione di Nefrologia

Istituti Ospitalieri, Cremona

f.malberti@ospedale.cremona.it

Nel centro ove presti attività qual è la percentuale di pazienti monitorati per CKD-MBD stadio 3-5 e quali marker biologici usi a tal fine?

Tutti i pazienti (circa 500) che afferiscono all'ambulatorio dellinsufficienza renale cronica (pazienti con CKD stadio 3-5) sono monitorati per le alterazioni del meta- bolismo del Ca-P. I pazienti, in relazione al filtrato e alla velocità di progessione della $\mathrm{CKD}$, hanno controlli ambulatoriali ogni 2-6 mesi. Gli esami includono sempre: calcemia, fosforemia, fosfaturia, azoturia (urine 24 ore). Il PTH, la 25 vitamina D plasmatica, le fosfatasi alcaline totali e ossee vengono in genere richiesti 2 volte l'anno.

Nel centro ove presti attività pratichi il dosaggio della $25(\mathrm{OH}) \mathrm{D}$ e/o della $1,25(\mathrm{OH})_{2} \mathrm{D} 3$ ?

Se si, in quale tipo di paziente e in quale percentuale? Se no, perché ritieni di non doverla praticare?

Dosiamo in tutti i pazienti con CKD 3-5 la 25 vitamina D plasmatica 1-2 volte l'anno. Non dosiamo di routine la 1,25 vitamina $\mathrm{D}$.

Applichi la correzione di 25(OH)D in caso di carenza? Perché, con quale protocollo ed in quale tipo di paziente? 
Attualmente non abbiamo un protocollo per la correzione del deficit di vitamina $\mathrm{D}$.

Esistono vari protocolli in letteratura che si sono mostrati validi nel correggere il deficit. Ne cito alcuni applicabili alla disponibilità in Italia (1-3):

Calcifediolo (Didrogyl) 3 gocce/die (pari a $15 \mu \mathrm{g}$ ), Colecalciferolo (vitamina D3) (Dibase, gocce): $1000 \mathrm{U} /$ die (= 4 gocce); oppure 30 gocce/settimana; oppure $1 \mathrm{f}$ da 100.000 U ogni 2 settimane, per 2 mesi, poi mensile.

Di fatto dato che nella CKD 4 anche normali livelli plasmatici di 25D3 non consentono di normalizzare i livelli plasmatici di 1-25D3, è più pratico l'uso di calcitriolo (0.25-0.50 $\mu \mathrm{g} / \mathrm{die})(1)$.

Nel centro ove presti attività in quale percentuale o in quali casi clinici particolari pratichi la biopsia ossea?

La biopsia ossea attualmente viene eseguita solo all'interno di studi clinici sperimentali. Negli ultimi anni sono state eseguite 5 biopsie in pazienti arruolati in un protocollo internazionale sull'uso del Cinacalcet (Studio BONAFIDE). Il problema principale delle biopsie ossee è la disponibilità di una corretta lettura istomorfometrica quantitativa. Vi sono pochi centri italiani in grado di eseguire tale lettura, di cui quello con maggiore esperienza nel settore nefrologico è l'Anatomia Patologica del Policlinico di Roma.

Nel centro ove presti attività in quale percentuale $e$ in quali stadi di CKD usi indagini strumentali per la ricerca delle calcificazioni vascolari? Quali indagini utilizzi?

Non abbiamo un protocollo che prevede la determinazione di uno score di calcificazione sulla base di indagini strumentali. Nei pazienti con CKD 3-5 le calcificazioni vascolari non vengono ricercate sistematicamente. Nei pazienti in dialisi vengono eseguiti (all'ingresso in dialisi e con frequenza di 1-2 anni): Rx ossa (colonna dorso-lombare, bacino, mani), Ecocardiogramma, Ecodoppler arterioso arti inferiori. Da questi esami si può ottenere un dato qualitativo sulla presenza delle calcificazioni vascolari.

Nel centro ove presti attività quale è la percentuale di pazienti in trattamento sostitutivo che resta nel range di normalità (secondo le linee guida SIN) per Ca, Pe PTH?

Nell'ultima valutazione effettuata (ottobre 2009) su 127 pazienti in dialisi extracorporea la distribuzione dei pazienti per i vari parametri era la seguente:
$\mathrm{P}>5.5 \mathrm{mg} / \mathrm{dL}$

$25 \%$ (23\% nel 2008)

$\mathrm{PTH}>300 \mathrm{pg} / \mathrm{mL}$

29\% (20\% nel 2008)

$\mathrm{PTH}<150 \mathrm{pg} / \mathrm{mL}$

$52 \%$ ( $61 \%$ nel 2008$)$

$\mathrm{Ca}>9.6 \mathrm{mg} / \mathrm{dL}$

$18 \%$ (22\% nel 2008)

La fosforemia media era $4.9 \pm 1.4 \mathrm{mg} / \mathrm{dL}$, il PTH medio era $268 \pm 310 \mathrm{pg} / \mathrm{mL}$.

Nel centro ove presti attività qual è la percentuale dei vari chelanti usati per ricercare il controllo del Ca-P nei pazienti in trattamento sostituitvo (anche in off-label treatment)?

Il 75\% dei pazienti in dialisi extracorporea usa sali di calcio, il 55\% il Sevelamer, il 10\% il carbonato di Lantanio. A nessun paziente vengono prescritti i sali di alluminio. L'associazione più frequente è basse dosi di sali di Ca associata a Sevelamer (25\% circa dei pazienti).

Nel centro ove presti attività qual è la percentuale di pazienti in CKD 3-5 sottoposta a dieta ipoproteica per il controllo della iperfosforemia?

La dieta ipoproteica non viene prescritta di routine. Alla maggior parte dei pazienti vengono dati consigli generici di limitare l'apporto proteico. L'uso di prodotti aproteici nelle fase più avanzata della $\mathrm{CKD}$ è frequente (circa il $20 \%$ dei pazienti).

Su 220 pazienti con CKD stadio 3-4 (eGFR medio $30 \mathrm{~mL} /$ $\mathrm{min})$ recentemente valutati, l'apporto proteico mediano (ricavato dall'escrezione urinaria di urea) era di $69 \mathrm{~g} / \mathrm{die}$ (circa $1 \mathrm{~g} / \mathrm{kg} / \mathrm{die}$ ). Questo dato indica che in almeno il $50 \%$ dei nostri pazienti sarebbe auspicabile una maggiore restrizione dell'apporto proteico.

Nel centro ove presti attività quale percentuale di pazienti in trattamento sostitutivo assumono vit-D o analoghi, calciomimetico, o entrambi?

Attualmente il 16\% dei pazienti in dialisi extracorporea è trattato con Cinacalcet. Il Cinacalcet è associato a metaboliti attivi della vitamina D nel $90 \%$ dei casi. Il $26 \%$ dei pazienti è in trattamento con calcitriolo o paricalcitolo ev. Il 5\% è in terapia con calcifediolo per os, il $31 \%$ assume calcitriolo per os. Complessivamente il $62 \%$ dei pazienti è trattato con metaboliti della vitamina D.

Dopo oltre un trentennio di osservazioni, esperienze, studi e ricerche, la fisiopatologia dell'iperparatiroidismo 
secondario non è ancora del tutto chiarita. Calcio, fosforo, recettori della vit-D o recettori sensibili del calcio, ormoni fosfatotropi e forse l'esistenza di un recettore cellulare del fosforo, sono tutti "in carriera" assieme al primo attore il PTH (almeno nella cronologia storica): si è in grado di prevedere un ordine e chiarimento delle cose o dobbiamo ancora e solo predire?

Il fattore emergente in questi ultimi anni nella fisiopatologia dell'iperparatiroidismo secondario (HPTs) è sicuramente il Fibroblast Growth Factor 23 (FGF-23). La scoperta di questo fattore ha consentito di chiarire alcuni aspetti patogenetici e ha portato alla rivalutazione del ruolo del $\mathrm{P}$ quale stimolo allo sviluppo dell'HPTs già nelle fasi iniziali della CKD. Il FGF-23 è considerato l'ormone che "contro-regola" la 1-25 vitamina $\mathrm{D}$, prevenendone gli effetti negativi, quali l'iperfosforemia, che possono insorgere in caso di ipersecrezione o elevato apporto esogeno (intossicazione da vitamina D). Il FGF23 è una proteina costituita da 251 aminoacidi con un peso molecolare di $26.000 \mathrm{Da}$. È prodotta dagli osteociti e viene degradata in 2 frammenti, il C-terminale (aa 180-251) e l'Nterminale (aa 1-24). I maggiori stimoli alla produzione di FGF-23 sono l'apporto dietetico di $\mathrm{P}$ e i livelli plasmatici di calcitriolo. A livello renale il FGF-23 ha due principali azioni: a) riduce l'attività del cotrasporto sodio-fosfato di tipo IIa (NaPi2a), determinando fosfaturia; b) inibisce l'attività della 1alfa-idrossilasi e stimola l'attività della 24-idrossilasi, riducendo così la sintesi di calcitriolo (4). Il più importante ruolo fisiologico del FGF-23 nei soggetti normali è quello di regolare l'escrezione renale di $\mathrm{P}$ in relazione all'apporto dietetico in modo da mantenere stabili i livelli di fosforemia. Infatti, l'aumento dell'apporto orale di P determina un aumento del FGF-23 nei soggetti normali $(4,5)$. Il FGF-23 aumenta precocemente nel corso della $\mathrm{CKD}$, come conseguenza della ritenzione di $\mathrm{P}$ dovuta alla riduzione dell'escrezione renale. Laumento del FGF-23 consente di mantenere normali i livelli plasmatici di $\mathrm{P}$ fino alle fasi più avanzate della $\mathrm{CKD}$, ma contribuisce alla riduzione dei livelli circolanti di 1-25 vitamina D $(4,6)$. Il deficit di calcitriolo stimola la secrezione di PTH contribuendo allo sviluppo dell'HPTs sia direttamente sia indirettamente (ipocalcemia conseguente alla riduzione dell'assorbimento intestinale di calcio).

Ovviamente con la scoperta del FGF-23 si sono chiariti alcuni importanti aspetti fisiopatologici dell'HPTs, ma rimangono ancora diversi punti oscuri. Gli aspetti che si stanno attualmente studiando, e il cui chiarimento potrebbe avere risvolti clinici interessanti in un prossimo futuro, sono quelli relativi ai feed-back "rene-intestino". Infatti, la recente documentazione che l'apporto dieteti- co di fosforo determina fosfaturia con un meccanismo d'azione rapido (minuti) indipendentemente da variazioni dei livelli plasmatici di calcio, fosforo, PTH e FGF23 (7), ha supportato l'ipotesi dell'esistenza di un asse entero-renale di regolazione dell'omeostasi del $\mathrm{P}$ e di un ormone (non ancora identificato) prodotto dall'intestino che agisce a livello renale inducendo fosfaturia. Inoltre, si sta cercando di chiarire il meccanismo attraverso il quale il P regola la produzione di FGF23 e il meccanismo con cui le cellule percepiscono le variazioni della fosforemia, non essendo ancora stato identificato un recettore cellulare per il fosforo.

Adynamic Bone Disease: una (relativamente) nuova entità classificativa o una patologia emergente? Sovrastimata o snobbata?

L'osteopatia adinamica (ABD) è diventata nell'ultimo decennio il tipo di osteodistrofia più frequente nei pazienti in dialisi, e in particolare in dialisi peritoneale. Nello studio di Sanchez et al (8), su 57 pazienti in dialisi peritoneale da 2-155 mesi sottoposti a biopsia ossea, il $63 \%$ aveva un quadro istologico di ABD. Il PTH medio nei pazienti con $\mathrm{ABD}$ era $89 \mathrm{pg} / \mathrm{mL}$ significativamente inferiore a quello dei pazienti con osteopatia ad alto turnover ( $358 \mathrm{pg} / \mathrm{mL}$ in media). I pazienti con $\mathrm{ABD}$, rispetto ai pazienti con osteopatia ad alto turnover, avevano una maggior frequenza di diabete ( $36 \%$ vs $14 \%$ ), erano più anziani, avevano assunto nell'ultimo anno maggiori dosi di sali di calcio ed erano trattati con maggior frequenza con soluzioni di dialisi ad alto contenuto di calcio. Gli Autori documentavano che livelli di PTH (IRMA-Nichols) $<150 \mathrm{pg} / \mathrm{mL}$ avevano un alto valore predittivo positivo (97\%) per ABD. Questo studio evidenziava che i principali fattori di rischio per osteopatia a basso turnover erano l'elevata concentrazione di calcio nel dialisato $(1,75$ $\mathrm{mmol} / \mathrm{L}$ ), le elevate dosi di sali di calcio, la presenza di diabete (l'iperglicemia inibisce la secrezione di PTH). Questi fattori determinerebbero l'ABD sopprimendo la secrezione di PTH. Altra causa nota di inibizione della sintesi del PTH è l'uso di elevate dosi di metaboliti attivi della vitamina D. Bisogna sottolineare che nell'uremia vi è resistenza ossea al PTH, per cui sono necessari livelli plasmatici di PTH più elevati del normale per mantenere un normale turnover osseo (9). Altre cause di ridotto turnover osseo nel paziente uremico sono l'intossicazione da alluminio, la malnutrizione e l'età avanzata. La malnutrizione determinerebbe riduzione del turnover osseo attraverso la riduzione del Insulinlike Growth Factor-I (IGF-I), che ha un'azione anabolica sull'osso (9).

L'interesse clinico per l'ABD deriva dai risultati di alcuni 
studi che hanno documentato un'associazione tra basso turnover osseo, sviluppo di calcificazioni vascolari e aumentata morbilità e mortalità $(10,11)$. Tuttavia, queste osservazioni hanno portato a una semplificazione eccessiva, per cui i bassi livelli di PTH sarebbero espressione di $\mathrm{ABD}$ e, quindi, comporterebbero un rischio aumentato di morbilità e mortalità. In realtà, a livelli di $\mathrm{PTH}<150$ $\mathrm{pg} / \mathrm{mL}$ spesso corrisponde un turnover osseo normale (12). Inoltre, linterpretazione è ancora più complessa e articolata, in quanto i pazienti con bassi livelli di PTH sono pazienti più anziani, spesso diabetici e malnutriti e con presenza di più comorbilità (11). Pertanto, il rischio non deriverebbe tanto dai livelli bassi di PTH quanto dall'elevata frequenza di comorbilità presente in questo gruppo di pazienti con bassi livelli di PTH (11). Diversi studi osservazionali su grosse casistiche hanno documentato che, dopo aggiustamento per vari fattori di rischio, i predittori di sviluppo di calcificazioni vascolari e di mortalità sono $\mathrm{i}$ livelli di PTH elevati piuttosto che i bassi livelli (13-16). Occorre puntualizzare che l'ABD è una condizione reversibile, una volta che si eliminano quei fattori responsabili della soppressione della sintesi del PTH. Lo studio di Haris et al (17) documenta che la riduzione della concentrazione di calcio nel dialisato è una misura efficace nel far regredire l'ABD. Infatti, nei 14 pazienti in DP con $\mathrm{ABD}$, in cui la concentrazione di calcio nelle soluzioni veniva ridotta da 1.625 a $1.0 \mathrm{mmol} / \mathrm{L}$, si aveva, dopo 16 mesi di follow-up, riduzione della calcemia, incremento del PTH nel range ottimale secondo le K/DOQI e normalizzazione del turnover osseo, che inizialmente era soppresso. Nel gruppo di controllo (mantenuto con concentrazione di calcio nel dialisato di $1.625 \mathrm{mmol} / \mathrm{L}$ ) il PTH non variava e il turnover osseo si manteneva soppresso. In sintesi, bassi livelli di PTH $(<150 \mathrm{pg} / \mathrm{mL})$ in assenza di intossicazione da alluminio sono suggestivi di turnover ridotto in circa il 50\% dei pazienti in dialisi (12). Non sono tanto i bassi livelli di PTH il "fattore di rischio" quanto le comorbilità frequentemente presenti nei pazienti che hanno bassi livelli di PTH (11). Tuttavia, la presenza di un basso turnover comporta una ridotta capacità dell'osso di tamponare un eventuale sovraccarico di calcio e fosforo, per cui in presenza di bassi livelli di PTH è consigliabile evitare un eccessivo apporto di calcio sia orale (chelanti del P contenenti $\mathrm{Ca}$ ) sia con il dialisato (bagni ad alta concentrazione di Ca). Lo studio DOPPS ha documentato, in più di 25.000 pazienti dializzati, come il rischio relativo di mortalità aumenti significativamente nei pazienti con bassi livelli di PTH se è contemporaneamente presente iperfosforemia (17).

Calciomimetici. Potenti, affidabili, indispensabili: quando, come e perché?
Un'elevata percentuale di pazienti dializzati ha livelli plasmatici di fosforo e PTH al di sopra dei target consigliati dalle linee guida K-DOQI. Uno studio recente ha dimostrato che i pazienti emodializzati incidenti che mantengono livelli plasmatici di calcio, fosforo e PTH in target nel primo anno di dialisi hanno un rischio di mortalità significativamente ridotto rispetto ai pazienti che hanno questi parametri alterati per diversi mesi nel corso dell'anno (18). Diversi studi randomizzati su grosse casistiche hanno dimostrato che nei pazienti dializzati affetti da HPTs (PTH $>300 \mathrm{pg} / \mathrm{mL}$ ) il Cinacalcet, rispetto alla terapia tradizionale con chelanti del $\mathrm{P}$ e metaboliti attivi della vitamina $\mathrm{D}$, consente un miglior controllo dei livelli di PTH (riduzione del PTH al di sotto di $300 \mathrm{pg}$ / mL nel 60-80\% dei casi a fine studio) e la contemporanea riduzione della calcemia e fosforemia (del 5-10\% rispetto ai livelli basali). Ne consegue che una maggior percentuale di pazienti affetti da HPTs raggiunge i target per il metabolismo calcio-fosforico se trattato con Cinacalcet (associato o meno a metaboliti attivi della vitamina D) rispetto a uno schema terapeutico che non preveda l'impiego di Cinacalcet. Inoltre, è stato dimostrato che con l'utilizzo di Cinacalcet si possono usare dosi più fisiologiche di metaboliti attivi della vitamina D con miglior controllo del metabolismo fosfo-calcico. È vero che a questi benefici sui parametri biochimici non corrisponde, a tutt'oggi, un dato probante in letteratura di effetti positivi su "hard-outcomes", quali la riduzione degli eventi cardiovascolari e della mortalità. A tal fine dovremo attendere i risultati di un grosso studio (EVOLVE) in cui sono stati arruolati più di 1000 pazienti emodializzati e che terminerà alla fine del 2011 . Fatte queste premesse, ritengo utile iniziare il trattamento con Cinacalcet in tutti quei pazienti dializzati che hanno livelli di PTH $>300$ $\mathrm{pg} / \mathrm{mL}$, nonostante il trattamento con dosi adeguate di metaboliti attivi della vitamina $\mathrm{D}$ (calcitriolo 1,5-3 $\mu \mathrm{g}$ / settimana, paracalcitolo 5-15 $\mu \mathrm{g}$ /settimana), e calcemia $>8.5 \mathrm{mg} / \mathrm{dL}$. La filosofia è quella di controllare l'HPTs prima che sia troppo avanzato utilizzando basse dosi di Cinacalcet e vitamina $\mathrm{D}$.

\section{Bibliografia}

1. Holick MF. Vitamin D deficiency. N Engl J Med 2007; 357: 266-81.

2. Jean G, Terrat JC, Vanel T, et al. High levels of serum fibroblast growth factors (FGF)-23 are associated with increased mortality in long haemodialysis patients. Nephrol Dial Transplant 2008; 23: 3670-6.

3. Courbebaisse M, et al. Effects of vitamin D supplementation on the calcium-phosphate balance in renal transplant 
patients. Kidney Int 2009; 75: 646-51.

4. Seiler S, Heine GH, Fliser D. Clinical relevance of FGF-23 in chronic kidney disease. Kidney Int 2009; 76 (Suppl 114): S34-42.

5. Nishida Y, Taketani Y, Yamanaka-Okumura H, et al. Acute effect of oral phosphate loading on serum fibroblast growth factor 23 levels in healthy men. Kidney Int 2006; 70: 2141-7.

6. Gutierrez O, Isakova T, Rhee E, et al. Fibroblastic growth factor 23 mitigates hyperphosphatemia but accentuates calcitriol deficiency in chronic kidney disease. J Am Soc Nephrol 2005; 16: 2205-15.

7. Berndt T, Thomas LF, Craig T, et al. Evidence for a signaling axis by which intestinal phosphate rapidly modulates renal phosphate reabsorption. Proc Nat Acad Sci USA 2007; 104: 11085-90.

8. Sanchez CM, Bajo MA, Selgas R, et al. Parathormone Secretion in Peritoneal Dialysis Patients With Adynamic Bone Disease. Am J Kidney Dis 2000; 36: 953.

9. Andress DL. Adynamic bone in patients with chronic kidney disease. Kidney Int 2008; 73: 1345-54.

10. London GM, Marty C, Marchais SJ, et al. Arterial calcifications and bone histomorphometry in end-stage renal disease. J Am Soc Nephrol 2004; 15: 1943-51.

11. Morrone LF, Mazzaferro S, Russo D, et al. Interaction between parathyroid hormone and the Charlson comorbidity index on survival of incident haemodialysis patients. Nephrol Dial Transplant 2009; 24: 2859-65.
12. Coen G, et al. Renal Osteodystrophy in Predialysis and Hemodialysis Patients: Comparison of Histologic Patterns and Diagnostic Predictivity of Intact PTH. Nephron 2002; 91: 103-11.

13. Coen G, Manni M, Mantella D, et al. Are PTH serum levels predictive of coronary calcifications in haemodialysis patients? Nephrol Dial Transplant 2007; 22: 3262-7.

14. Young EW, Albert JM, Satayathum S, et al. Predictors and consequences of altered mineral metabolism: the Dialysis Outcomes and Practice Patterns Study. Kidney Int 2005; 67: 1179-87.

15. Melamed ML Eustace JA, Plantinga L, et al. Changes in serum calcium, phosphate, and PTH and the risk of death in incident dialysis patients: a longitudinal study. Kidney Int 2006; 70: 351-7.

16. Tentori F, Blayney MJ, Albert JM, et al. Mortality risk for dialysis patients with different levels of serum calcium, phosphorus, and PTH: The Dialysis Outcomes and Practice Patterns Study (DOPPS). Am J Kidney Dis 2008; 52: 519-30.

17. Haris A, Sherrard DJ, Hercz G. Reversal of adynamic bone disease by lowering of dialysate calcium. Kidney Int 2006; 70: 921-7.

18. Danese MD Belozeroff V, Smirnakis K, Rothman KJ. Consistent control of mineral and bone disorder in incident hemodialysis patients. Clin J Am Soc Nephrol 2008; 3: 1423-9.

\section{Intervista a Domenico Russo}

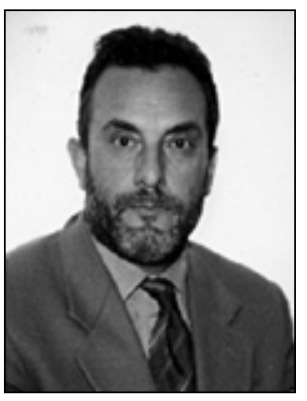

Dr. Domenico Russo

Prof. Associato di Nefrologia

Dipartimento di Patologia

Sistematica

Università Degli Studi

di Napoli "Federico II"

domenicorusso51@hotmail.com

Nel centro ove presti attività qual è la percentuale di pazienti monitorati per CKD-MBD stadio 3-5 e quali marker biologici usi a tal fine?

La popolazione con CDK 2-5 non in follow- up presso la mia area funzionale rappresenta il 65-70\%. Indagini biochimiche generali (con fibrinogeno, omocisteina, PCR) con PTH, e fosfatasi alcalina controllati ogni 3-6 mesi a seconda dello stadio CKD.

Nel centro ove presti attività pratichi il dosaggio della $25(\mathrm{OH}) \mathrm{D}$ e/o della $1,25(\mathrm{OH})_{2} \mathrm{D} 3$ ?

Se si, in quale tipo di paziente e in quale percentuale? Se no, perché ritieni di non doverla praticare?

Ritengo il dosaggio della $25 \mathrm{OH}$ importante per i pazienti con CKD (da effettuare almeno due volte l'anno). Nella mia struttura non è un esame previsto dal servizio centralizzato per cui non la doso.

Applichi la correzione di $25(\mathrm{OH}) D$ in caso di carenza? Perché, con quale protocollo e in quale tipo di paziente?

Non dosando la 25oh la somministrazione di substrato della vitamina $D$ viene effettuata su considerazioni strettamente clinico-laboratoristiche.

Nel centro ove presti attività in quale percentuale o in quali casi clinici particolari pratichi la biopsia ossea? 
Non viene praticata la biopsia ossea.

Nel centro ove presti attività in quale percentuale $e$ in quali stadi di CKD usi indagini strumentali per la ricerca delle calcificazioni vascolari? Quali indagini utilizzi?

La valutazione delle calcificazioni vascolari viene effettuata nell' $80 \%$ dei pazienti con CKD mediante CT multistrato oppure con Rx diretto laterale della colonna lombare (metodo di Kauppila).

Nel centro ove presti attività qual è la percentuale di pazienti in trattamento conservativo che resta nel range di normalità (secondo le linee guida SIN) per Ca, Pe PTH?

L'80-90\% dei pazienti in stadio 3-4 CKD non presenta alterazioni delle variabili del metabolismo minerale. La percentuale scende al $60-70 \%$ nei pazienti con stadio 5 CKD.

Nel centro ove presti attività qual è la percentuale dei vari chelanti usati per ricercare il controllo del Ca-P nei pazienti in trattamento conservativo (anche in off-label treatment)?

$10-15 \%$.

Nel centro ove presti attività qual è la percentuale di pazienti in CKD 3-5 sottoposta a dieta ipoproteica per il controllo della iperfosforemia?

10-15\% dei pazienti in stadio $3 \mathrm{CKD}$ necessitano di restrizione proteica per il controllo della fosforemia; la percentuale sale al $50 \%$ e al $70 \%$ rispettivamente negli stadi 4 e $5 \mathrm{CKD}$. Bisogna tener conto che una restrizione proteica è necessaria non sempre per ottenere il controllo della fosforemia.

Nel centro ove presti attività qual è la percentuale di pazienti in trattamento conservativo che assumono vitamina $D$ o analoghi?

$10 \%$ e prevalentemente per i pazienti in stadio $5 \mathrm{CKD}$.

Il fenomeno detto "epidemiologia inversa" tipico del paziente con CKD in trattamento sostitutivo, non vede rispettata la classica relazione tra ipertensione arteriosa ed eventi cardiovascolari. Quale spiegazione per tale fenomeno e quale ruolo gioca nella $C K D-M B D$ ?

Numerosi studi riportano che, in contrasto alla popolazione generale dove la supernutrizione si associa a un aumentato rischio di malattia cardiovascolare, nei pazienti in dialisi una riduzione del BMI o una riduzione del colesterolo o della creatinina è fortemente associata ad aumentata morbilità e mortalità per eventi cardiovascolari. Risultati simili sono riportati per la pressione arteriosa; nei pazienti in dialisi la bassa pressione arteriosa più che l'ipertensione arteriosa sembra essere associata ad eventi cardiovascolari avversi. Questo effetto paradosso è definito "epidemiologia inversa" oppure "fattore di rischio paradosso". Questa terminologia non vuole indicare che nella popolazione in dialisi sussistono meccanismi patofisiologici differenti per quanto riguarda gli effetti sia della nutrizione sia dell'ipertensione arteriosa rispetto ad altre popolazioni. Un basso BMI può indicare una malnutrizione primitiva, oppure dovuta ad altre patologie concomitanti (epatiche, gastro-intestinali, malattie sistemiche) oppure secondaria alla infiammazione cronica della "uremia". Una bassa pressione arteriosa può ben essere dovuta a una patologia cardiaca cronica a bassa gittata responsabile del successivo evento fatale.

Situazioni cliniche del tipo in precedenza descritte concorrono sicuramente all'elevata mortalità che caratterizza tutti i pazienti nei vari stadi della CKD. Quanto l'epidemiologia inversa sia presente nella CKD-MBD può essere indicato dalla fosforemia. Nel paziente in dialisi una bassa fosforemia $(<3 \mathrm{mg} / \mathrm{dL})$ si associa a un elevato rischio di mortalità sia per tutte le causa che per quelle cardiovascolari. E verosimile che la bassa fosforemia sia un marker di malnutrizione, presenza di comorbidità, ed infiammazione cronica. Allo stesso modo un PTH ai limiti bassi del range di normalità del laboratorio di riferimento può associarsi a una più alta mortalità dovuta e un eccessivo intervento di normalizzazione dei parametri del metabolismo minerale.

PTH e CKD-MBD: quanto artefice o quanto vittima? Ad esempio la carenza di vitamina $D$ (o meglio la riduzione dell'attivazione dei suoi recettori nucleari) è sicuramente un evento che precede l'iperparatiroidismo secondario. Quale di questi due giocatori è in "fuori gioco" per lo sviluppo della patologia ossea dell'uremico e a quale dei due - se possibile - si può imputare un predominio sullo sviluppo di CKD-MBD?

E stato documentato che la riduzione delle concentrazioni sia di 25-OH che di calcitriolo compare sin dalle fasi precoci della CKD. Il PTH aumenta per correggere la conseguente ipocalcemia per un'azione sul rene, sull'intestino e sull'osso. Altri fattori che 
concorrono alliper-PTH secondario sono fosforemia e FGF-23 (sempre stimolato dalla fosforemia). Il controllo del metabolismo minerale dovrebbe essere considerato come un sistema integrato nel quale ogni componente svolge un ruolo critico. Non ci sono "primi attori" e "comparse". Il singolo componente può avere un maggiore o minore peso oppure essere "artefice e vittima" in funzione dello stadio CKD.

Fosforo chiama "ormone"! Rispondono PTH, FGF23 e Klotho mentre 1,25(OH) $D 3$ resta a guardare. Una relazione pericolosa? Più di un triangolo (fisiopatologico)? Un paradigma o cosa?

In condizioni fisiologiche il fosforo induce la produzione di FGF-23 dagli osteociti; l'ormone riduce il riassorbimento tubulare di fosforo normalizzando la concentrazione sierica dello ione. L'azione fosfaturica di FGF-23 sembra essere più efficace di quella espressa dal PTH. In presenza di massa nefronica funzionante ridotta, come avviene in $\mathrm{CKD}$, la fosforemia non può essere più corretta mediante l'aumento della fosfaturia da parte dei due ormoni. Permangono, però, tutti gli altri effetti negativi e contrapposti di FGF-23 e PTH. Il primo, infatti, inibendo la 1-a idrossilasi (quindi la concentrazione di calcitriolo) stimola la produzione di PTH per la conseguente ipocalcemia. Un alto PTH riduce la secrezione di FGF-23 ma ha effetti negativi sull'osso.

Vitamina D e suoi precursori sembrano avere un ruolo "passivo"; essi "subiscono" eventi esterni. Se vista alla luce della riduzione della attivazione dei recettori nucleari, sicuramente la vitamina $\mathrm{D}$ non è una comparsa sullo scenario delle alterazioni del metabolismo minerale.

Nonostante tutto (linee guida comprese, SIN, K/DOKI, KDIGO) nella maggioranza dei casi i target terapeutici per calcio, fosforo e PTH non vengono centrati e soprattutto mantenuti. Quale commento ti stimola tale insufficiente raggiungimento?

Sia i dati dello studio DOPPS (in tutte le sue tornate) che quelli dello studio KEEP, hanno chiaramente evidenziato che nei pazienti in trattamento sostitutivo il controllo delle variabili del metabolismo minerale rimane insoddisfacente a distanza di diversi anni dalla pubblicazione delle linee guida K-DOQI. Il controllo del fosforo si ottiene mediamente nel $50 \%$ della popolazione in dialisi; il fosforo è prevalentemente alto nella restante parte della popolazione. Il controllo del PTH è ancora meno soddisfacente con una tendenza a un basso PTH nei pazienti che non sono a target. I dati del DOPPS relativi ai pazienti in sostitutiva in Italia rispecchiano in grande misura i dati del DOPPS generale.

Non si hanno delle spiegazioni definitive per tali risultati non brillanti, malgrado la migliore conoscenza della fisiopatologia delle alterazioni del metabolismo minerale, la diffusione delle linee guida, eventiscientifici, disponibilità difarmacimolto efficaci. E verosimile che i non brillanti risultati possano essere imputati a un approccio terapeutico "settoriale" dei componenti delle complesse alterazioni del metabolismo minerale. In un sistema integrato, quale è il metabolismo minerale, la correzione di un solo componente del sistema non porta al reset delle alterazioni. L'impiego concomitante di più farmaci che incidono su punti diversi di un metabolismo minerale alterato potrebbe migliorare la percentuale dei pazienti a target per calcio, fosforo e PTH.

\section{Commento conlcusivo}

Le regolazione del metabolismo minerale risponde a meccanismi fisiologici ben precisi e delineati nelle loro caratteristiche negli ultimi anni. Più recentemente l'attenzione del Nefrologo si è concentrata sul ruolo di FGF-23 nella regolazione del fosforo. É opinione dell'Autore che questo ormone meriti notevole attenzione per il suo ruolo cruciale nella regolazione della fosforemia soprattutto nelle fasi iniziali dell'insufficienza renale. I dati acquisiti indicano che FGF-23 può rappresentare il marcatore precoce delle alterazioni del metabolismo minerale.

FGF-23 è un nuovo ormone che regola l'omeostasi del fosforo. Esso viene prodotto dagli osteociti e osteoblasti dalla fosforemia. FGF-23 riduce il riassorbimento tubulare prossimale e di conseguenza i livelli sierici del fosforo, inibendo un co-trasportatore sodio-dipendente presente sull'orletto a spazzola. La regolazione della fosforemia da parte di FGF 23 avviene anche attraverso l'inibizione della 1-a idrossilasi che comporta una ridotta produzione di calcitriolo; il riassorbimento intestinale di fosforo e di calcio si riduce. L'effetto sulla fosfaturia ha indubbiamente ricadute positive per il paziente; non altrettanto positivi sono gli effetti del ridotto assorbimento di calcio. L'ipocalcemia stimola la produzione del PTH, inducendo un peggioramento dell'iperPTH secondario.

FGF 23 è molto elevato nei pazienti in dialisi ed è stato posto in relazione all'alta mortalità osservata 
in questa popolazione. L'associazione con la mortalità risulta essere indipendente da altri fattori di rischio. Pazienti con FGF 23 più alto hanno un rischio di mortalità per tutte le cause 600 volte maggiore. Diverse ipotesi sono state proposte per spiegare l'associazione tra FGF-23 ed alto rischio di mortalità. L'effetto negativo sulla 1-a idrossilasi e ridotta produzione di calcitriolo potrebbe essere un'ipotesi. Alto FGF-23 è associato a ridotta efficacia della terapia con calcitriolo e quindi a un più marcato iperparatiroidismo secondario che può causare alta mortalità. Alto FGF-23 è stato associato a ipertrofia ventricolare sinistra che può essere responsabile dell'elevata incidenza di eventi cardiovascolari. I meccanismi attraverso cui FGF-23 agisce sui cardiomiociti non sono stati ancora definiti.

Nei pazienti in dialisi, quindi, FGF-23, stimolato dalla fosforemia, induce una cascata di eventi tutti nefasti. Differente, invece, il ruolo dell'ormone nelle fasi iniziali della insufficienza renale dove potrebbe svolgere un ruolo "parzialmente" protettivo. FGF-23 aumenta in proporzione alla riduzione della funzione renale. Il suo incremento si osserva anche in presenza di fosforemia nei range di normalità attualmente accettati. In questa fase FGF-23 controlla la fosforemia aumentando l'escrezione urinaria dello ione.
A fronte di questa azione "protettiva" restano tuttavia le azioni negative sulla 1-a idrossilasi e calcitriolo.

$\grave{E}$ utile dosare FGF-23?

Nel paziente in dialisi la risposta più ragionevole è no. E certo che l'ormone è alto e quindi va controllata con estrema attenzione la fosforemia mediante dieta e chelanti del fosforo da una parte e utilizzando con cautela vitamina D e suoi analoghi per gli effetti sia diretti che indiretti che essi hanno sulla secrezione di FGF-23.

Quale comportamento assumere nel paziente in terapia conservativa?

Probabilmente in questa popolazione il dosaggio di FGF-23 è meritevole di considerazione. Un'alta concentrazione sierica dell'ormone potrebbe indicare una situazione di iperfosfatemia non evidenziabile con il dosaggio della sola fosforemia e degli altri parametri del metabolismo minerale.

FGF-23 sottolinea e conferma che tuttora la fosforemia gioca un ruolo fondamentale nelle alterazioni del metabolismo minerale in tutti gli stadi della CKD. 